\title{
Photonic Jackiw-Rebbi states in all-dielectric structures controlled by bianisotropy
}

\author{
Alexey A. Gorlach, ${ }^{1, *}$ Dmitry V. Zhirihin, ${ }^{2}$ Alexey P. Slobozhanyuk, ${ }^{2,3}$ \\ Alexander B. Khanikaev, ${ }^{2,4,5}$ and Maxim A. Gorlach ${ }^{2, \dagger}$ \\ ${ }^{1}$ Belarusian State University, Minsk 220030, Belarus \\ ${ }^{2}$ ITMO University, Saint Petersburg 197101, Russia \\ ${ }^{3}$ Nonlinear Physics Centre, Research School of Physics and Engineering, Australian National University, \\ Canberra ACT 2601, Australia \\ ${ }^{4}$ Department of Electrical Engineering, Grove School of Engineering, City College of the City University of New York, \\ NY 10031, USA \\ ${ }^{5}$ Graduate Center of the City University of New York, NY 10016, USA
}

(Received 25 November 2018; published 14 May 2019)

\begin{abstract}
Electric and magnetic resonances of dielectric particles have recently uncovered a range of exciting applications in light steering at the nanoscale. Breaking of particle inversion symmetry further modifies its electromagnetic response, giving rise to bianisotropy known also as magnetoelectric coupling. Recent studies suggest the crucial role of magnetoelectric coupling in realization of photonic topological metamaterials. To further unmask this fundamental link, we design and test experimentally one-dimensional array composed of dielectric particles with overlapping electric and magnetic resonances and broken mirror symmetry. Flipping over half of the meta-atoms in the array, we observe the emergence of interface states providing photonic realization of the celebrated Jackiw-Rebbi model. We trace the origin of these states to the fact that local modification of particle bianisotropic response affects its effective coupling with the neighboring meta-atoms, which provides a promising avenue to engineer topological states of light.
\end{abstract}

DOI: 10.1103/PhysRevB.99.205122

\section{INTRODUCTION}

Electric and magnetic resonances supported by dielectric particles $[1,2]$ have initiated an entire direction of alldielectric nanophotonics, opening a route toward metamaterials and metadevices without lossy plasmonic components [3,4] featuring unique functionalities both in linear [5] and nonlinear regimes [6]. Bianisotropy, which stems from the particle symmetry reduction, further broadens the plethora of available physical effects enabling the coupling between electric and magnetic responses of a particle.

An important application of bianistropic meta-atoms is associated with engineering of time-reversal-invariant photonic topological metamaterials $[7,8]$. In contrast to the majority of systems, photonic topological structures support electromagnetic modes which possess a unique property of spin-locked unidirectional propagation along the boundaries. Besides that, photonic topological states feature considerable robustness against disorder or imperfections, which is of paramount importance for applications [9-12]. Recently, experimental works have demonstrated photonic topological structures in two $[13,14]$ and three [15] spatial dimensions with topological states facilitated by magnetoelectric coupling.

The next conceptual step, in our opinion, is related to the analysis of photonic structures with spatially varying bianisotropic response, which may give rise to even richer variety of topologically protected states. In this paper, aiming

\footnotetext{
*alexey.gorlach@gmail.com

${ }^{\dagger}$ m.gorlach@metalab.ifmo.ru
}

to further unmask the fundamental link between bianisotropy and topological states of light, we investigate an array composed of dielectric meta-atoms with broken mirror symmetry and different bianisotropic response [Fig. 1(a)]. Based on the discrete dipole approximation, we derive an effective photonic Hamiltonian for such system and demonstrate the interface states at the boundary between the two halves of the array with the different signs of magnetoelectric coupling. Such modes are proved to be photonic analogues of Jackiw-Rebbi states which have recently been investigated in various systems including transmission line metamaterials [16], driven slowlight systems [17] and waveguide arrays [18].

The key result of our analysis is that local modification of the disk bianisotropy translates into the modification of coupling constants in the effective photonic Hamiltonian. Apparently, this opens a route to engineer electromagnetic topological states via the staggered bianisotropy pattern. To confirm our findings, we realize such one-dimensional array experimentally [Fig. 1(b)], proving the emergence of the interface states by mapping the near fields of the structure.

\section{THEORETICAL MODEL}

To grasp the physics of the proposed 1D system, we develop a theoretical model based on the discrete dipole approximation [19-22]. To simplify the treatment, we take into account only $x$ and $y$ components of electric and magnetic dipole moments of the disks neglecting off-resonant $z$ components along with higher-order multipoles. Additionally, we consider interaction of the disk with its nearest neighbors 


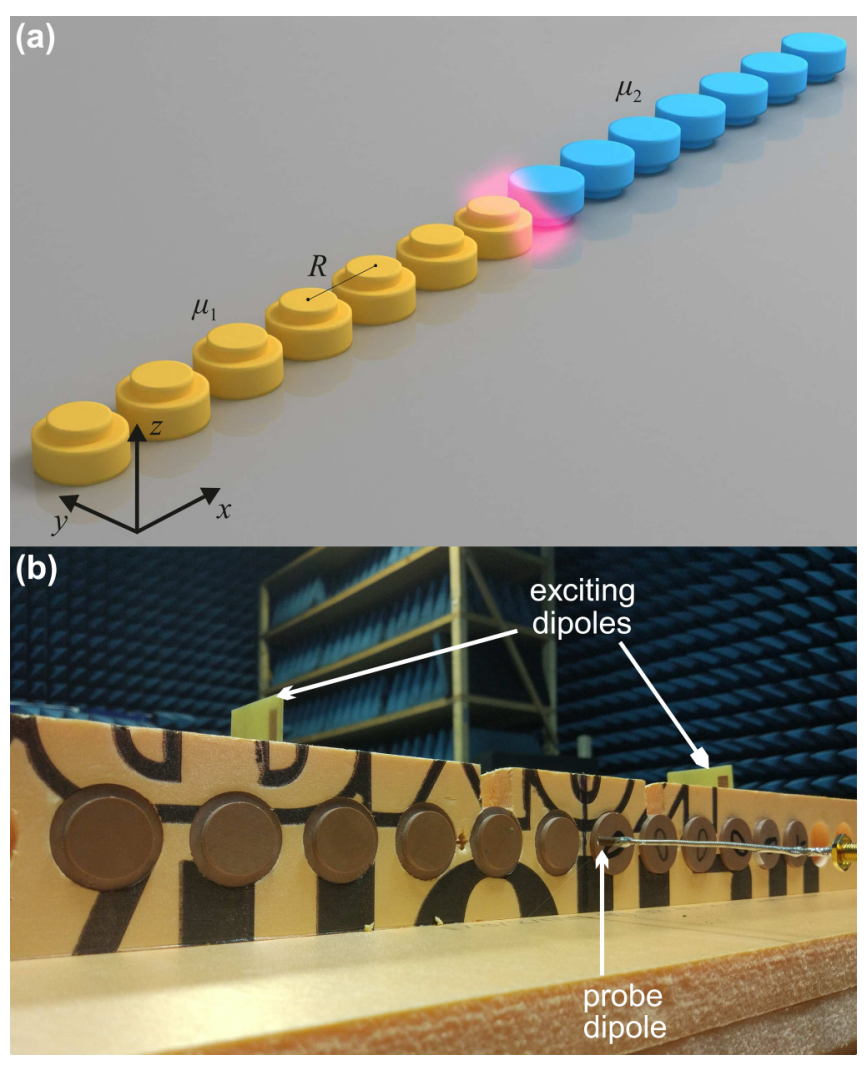

FIG. 1. One-dimensional array composed of disks with different magnitudes of bianisotropy parameters: $\mu_{1}$ and $\mu_{2}$. (a) A schematic of the structure. (b) Photograph of experimental sample composed of $N=12$ disks.

only. This yields the set of equations:

$$
\begin{aligned}
& \left(\begin{array}{cc}
\hat{\alpha}^{\mathrm{ee}} & \hat{\alpha}^{\mathrm{em}} \\
\hat{\alpha}^{\mathrm{me}} & \hat{\alpha}^{\mathrm{mm}}
\end{array}\right)^{-1}\left(\begin{array}{c}
\mathbf{p}_{\mathbf{n}} \\
\mathbf{m}_{n}
\end{array}\right) \\
& \quad=\sum_{l=n \pm 1}\left(\begin{array}{cc}
\hat{G}^{\mathrm{ee}}\left(\mathbf{r}_{n l}\right) & \hat{G}^{\mathrm{em}}\left(\mathbf{r}_{n l}\right) \\
\hat{G}^{\mathrm{me}}\left(\mathbf{r}_{n l}\right) & \hat{G}^{\mathrm{mm}}\left(\mathbf{r}_{n l}\right)
\end{array}\right)\left(\begin{array}{c}
\mathbf{p}_{\mathbf{l}} \\
\mathbf{m}_{l}
\end{array}\right) .
\end{aligned}
$$

Here, $\hat{G}(\mathbf{r})$ is the dyadic Green's function describing electric and magnetic fields produced by electric and magnetic dipoles as labelled by the superscripts ee, em, me and $\mathrm{mm}$ [23]; $\mathbf{p}$ and $\mathbf{m}$ are the two-component vectors containing $x$ and $y$ components of electric and magnetic dipole moments, respectively; $\hat{\alpha}^{\mathrm{ee}}, \hat{\alpha}^{\mathrm{mm}}$ are tensors of electric and magnetic polarizability of the disk, and off-diagonal tensors $\hat{\alpha}^{\mathrm{em}}$ and $\hat{\alpha}^{\mathrm{me}}$ describe the effect of magnetoelectric coupling.

Rotational symmetry of the disk with respect to the $O z$ axis and mirror symmetry in the $O x z$ plane impose constraints on the structure of the polarizability tensors: $\hat{\alpha}^{\text {ee }}$ and $\hat{\alpha}^{\mathrm{mm}}$ are proportional to identity matrix, while the tensors of magnetoelectric coupling read

$$
\hat{\alpha}^{\mathrm{em}}=\hat{\alpha}^{\mathrm{me}}=\left(\begin{array}{cc}
0 & i \chi \\
-i \chi & 0
\end{array}\right) .
$$

Moreover, by the proper choice of the disks parameters, we can ensure equal magnitudes of electric and magnetic polarizabilities in the frequency range of interest: $\hat{\alpha}^{\mathrm{ee}}=\hat{\alpha}^{\mathrm{mm}}=\beta \hat{I}$.
Further details on the analysis of this model are provided in the Supplemental Material [24], Secs. 1 and 2.

The inverted $4 \times 4$ polarizability tensor takes the form

$$
\left(\begin{array}{cccc}
u & 0 & 0 & -i v \\
0 & u & i v & 0 \\
0 & -i v & u & 0 \\
i v & 0 & 0 & u
\end{array}\right)
$$

where $u=\beta /\left(\beta^{2}-\chi^{2}\right)$ and $v=\chi /\left(\beta^{2}-\chi^{2}\right)$. Additionally, in the vicinity of the disk resonance $\omega_{0}$, we approximate the frequency dependence of $u$ as $\left(\omega-\omega_{0}\right) / A$, which corresponds to the pole approximation of polarizability. With these assumptions, Eq. (1) can be reformulated as an eigenvalue problem with the effective Hamiltonian and the "wave function":

$$
\hat{H}|\psi\rangle=E|\psi\rangle \text {. }
$$

For convenience, we use eigenmodes of an isolated disk as a basis, in which case the wave function is defined in terms of electric and magnetic dipole moments as follows:

$$
|\psi\rangle=\left(p_{x}+i m_{y}, p_{x}-i m_{y}, p_{y}-i m_{x}, p_{y}+i m_{x}\right)^{T},
$$

and normalized to unity as usual, and "energy" parameter is related to the frequency of the mode $E=2\left(\omega-\omega_{0}\right) R^{3} / A$ such that zero energy corresponds to the frequency of dipole resonance of an isolated disk. The effective photonic Hamiltonian takes the form

$$
\hat{H}=\left(\begin{array}{cc}
\hat{H}_{\mathrm{s}}^{(+)} & 0 \\
0 & \hat{H}_{\mathrm{s}}^{(-)}
\end{array}\right),
$$

where each of the $2 \times 2$ blocks is given by the expression

$$
\hat{H}_{\mathrm{s}}^{( \pm)}=2 \hat{I} \cos k+\mu \sigma_{z} \pm 6 \sigma_{x} \cos k,
$$

$\mu=2 v R^{3}$ and $\sigma_{i}$ stand for Pauli matrices. We interpret two blocks of the Hamiltonian as two effective pseudospins. Spin-up configuration $\left|\psi_{+}\right\rangle$corresponds to the nonzero $p_{x}$ and $m_{y}$ components of dipole moments, whereas spin-down configuration $\left|\psi_{-}\right\rangle$is characterized by the nonzero $p_{y}$ and $m_{x}$. Essentially, energy spectra for two pseudospins coincide and read

$$
E=2 \cos k \pm \sqrt{\mu^{2}+36 \cos ^{2} k}
$$

Energy spectrum calculated from Eq. (7) is shown in Fig. 2. In the case $\mu=0$, we observe two crossing points $k_{1,2}=\mp \pi / 2$. In the vicinity of these points $k=k_{1,2}+\delta k$, the dispersion is linear $E_{a}=\mp 4 \delta k$ and $E_{b}= \pm 8 \delta k$. At the same time, the Hamiltonian Eq. (7) takes the form of type-I Dirac Hamiltonian [25],

$$
\hat{H}_{\mathrm{s}}^{( \pm)}=2 \delta k \tau_{z}+\mu \sigma_{z} \pm 6 \sigma_{x} \tau_{z} \delta k,
$$

where the mass term $\mu=2 v R^{3}$ quantifies magnetoelectric coupling in the disk and elements of Pauli matrix $\tau_{z}$ label inequivalent points in reciprocal space: $\tau_{z}=1$ for $k_{1}=-\pi / 2$ and $\tau_{z}=-1$ for $k_{2}=\pi / 2$. In analogy to the standard Dirac Hamiltonian [26], this model features zero-energy JackiwRebbi states at the boundary of the domains with the opposite sign of the mass term (see Supplemental Material [24] Sec. 3.1).

Since there are two Dirac points in the spectrum, $k_{1}=$ $-\pi / 2$ and $k_{2}=\pi / 2$, the Hamiltonian Eq. (9) features two 


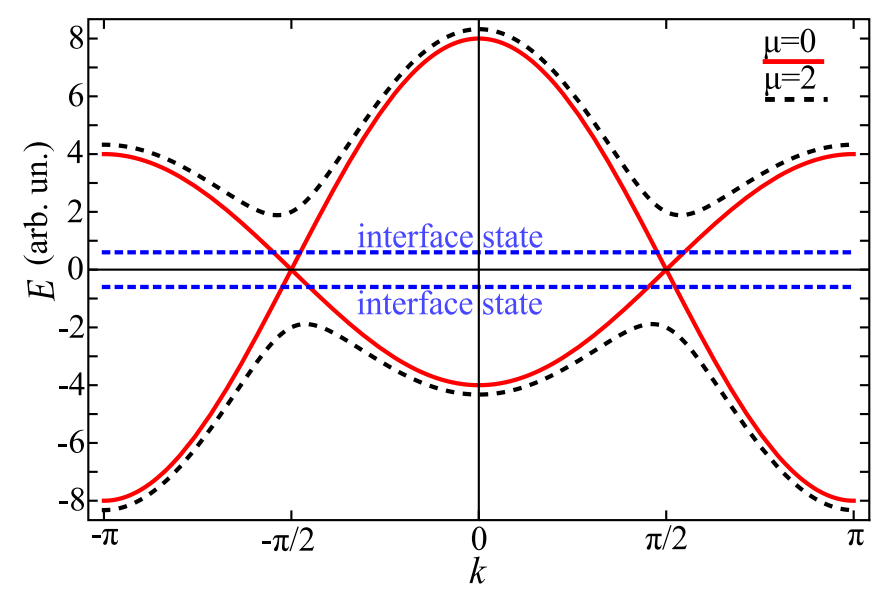

FIG. 2. The dispersion of dipole excitations $E(k)$ for the infinite array without and with bianisotropy (red solid and black dashed lines, respectively). There are two Dirac points at $k_{1,2}=\mp \pi / 2$. Dashed blue lines indicate the energy of the interface states which appear in the presence of the domain wall.

interface states. Furthermore, each of these states is doubly degenerate due to the polarization degree of freedom, and hence we expect two doublets of polarization-degenerate interface states. While the existence of the interface states can be inferred from the simplified type-I Dirac Hamiltonian, correct evaluation of their energies requires more involved calculation, which is done in Sec. 3.2 of the Supplemental Material [24]. The symmetries of the system ensure that the splitting of interface states is always symmetric with respect to zero energy (Supplemental Material [24] Sec. 3.3), whereas the magnitude of splitting is governed by the dimensionless parameter $\mu$.

In particular, in the limit of weak magnetoelectric coupling $\mu \ll 1$, the energies of the interface states can be approximated by $E= \pm \mu^{2} / 6$, whereas for larger $\mu$, the interface states are pushed away from the band-gap center. However, they do not disappear in the continuum of bulk states even for extremely large values of magnetoelectric coupling $\mu \gg 1$.

The results of the presented analytical model exhibit good qualitative agreement with full-wave numerical simulations carried out in Comsol Multiphysics (Fig. 3). The simulations reveal in particular that the spectrum of bulk excitations in the array has a band gap, which hosts four states inside it. These four states are grouped into two doublets positioned symmetrically with respect to the band-gap center. The calculated mode profiles, shown as insets in Fig. 3, suggest that the field for these four states is indeed localized at the domain wall.

\section{ORIGIN OF THE INTERFACE STATES: MODIFICATION OF COUPLING DUE TO BIANISOTROPY}

To further clarify the origin of the interface states in our model, it is instructive to examine the limit of strong bianisotropy $\mu \gg 1$. We analyze geometry in Fig. 4(a), in which case periodicity of the array is broken by the domain wall.

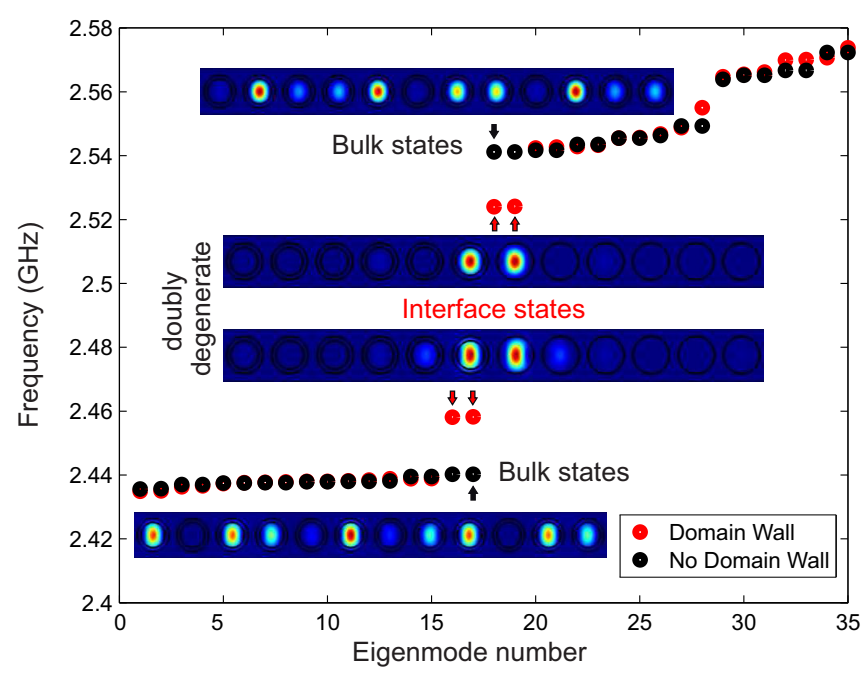

FIG. 3. Eigenmodes of the array composed of ceramic metaatoms with permittivity $\varepsilon=39$ calculated with Comsol Multiphysics software. In numerical simulation, the array is placed in a cylindrical waveguide of the radius $r_{w}=21.3 \mathrm{~mm}$ with perfectly conducting walls. Periodic boundary conditions along $x$ axis are applied. One period contains 16 particles. Each meta-atom consists of two closely attached disks with diameters $29.1 \mathrm{~mm}$ and $22.0 \mathrm{~mm}$ and heights $9.0 \mathrm{~mm}$ and $3.0 \mathrm{~mm}$, respectively. Distance between the centers of the neighboring disks is $R=36 \mathrm{~mm}$. Insets show the calculated distribution of magnetic field $\left|H_{x}\right|^{2}+\left|H_{y}\right|^{2}$ for bulk and interface modes. Note that interaction of the array with the waveguide causes small blueshift of all eigenmodes in comparison with the experiment performed in an anechoic chamber.

Therefore, Eqs. (6) and (7) have to be replaced by

$$
\begin{aligned}
E\left|\varphi_{n}\right\rangle= & \left(\begin{array}{cc}
\mu_{n} & 0 \\
0 & -\mu_{n}
\end{array}\right)\left|\varphi_{n}\right\rangle \\
& +\left(\begin{array}{ll}
1 & t \\
t & 1
\end{array}\right)\left(\left|\varphi_{n-1}\right\rangle+\left|\varphi_{n+1}\right\rangle\right),
\end{aligned}
$$

where the same basis Eq. (5) is used, and $|\varphi\rangle=\left(a_{n}, b_{n}\right)^{T}$ denotes a two-component wave function for the single pseudospin, where $a_{n}=p_{n x}+i m_{n y}$ and $b_{n}=p_{n x}-i m_{n y}$ for spinup configuration. $t= \pm 3$ for up and down pseudospins, respectively.

Now consider a fixed band with energy $E \approx \mu$. In our geometry, $\mu_{n}=\mu$ for $n<0$ and $\mu_{n}=-\mu$ for $n>0$ [Fig. 4(a)]. Then, Eq. (10) suggests that the amplitudes $b_{n}$ for $n<0$ and $a_{n}$ for $n>0$ scale as $1 / \mu$ and can be neglected in the limit

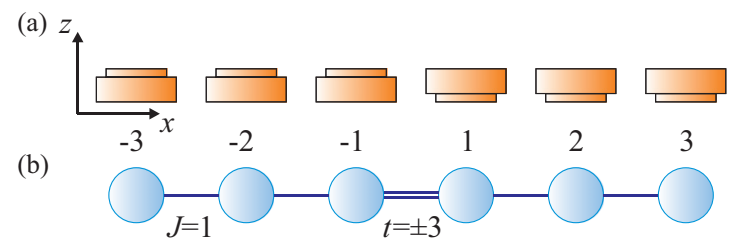

FIG. 4. Mapping of electromagnetic problem (a) onto the tightbinding model, (b) valid in the limit of strong bianisotropy $\mu \gg 1$. Note that local bianisotropy of meta-atoms controls the magnitude of couplings in the effective tight-binding model. 
$\mu \gg 1$. Truncating the system Eq. (10) to include only the leading-order amplitudes $a_{n}$ for $n<0$ and $b_{n}$ for $n>0$, we get

$$
\begin{gathered}
(E-\mu) a_{n}=a_{n-1}+a_{n+1}(n \leqslant-2), \\
(E-\mu) a_{-1}=a_{-2}+t b_{1}, \\
(E-\mu) b_{1}=t a_{-1}+b_{2}, \\
(E-\mu) b_{n}=b_{n-1}+b_{n+1}(n \geqslant 2) .
\end{gathered}
$$

In turn, the system of Eqs. (11)-(14) corresponds precisely to the tight-binding model Fig. 4(b), which provides a simple interpretation of predicted interface states. Furthermore, this simplified model yields the energy of the interface state, $E=\mu-10 / 3$, which agrees with the limiting case $\mu \gg 1$ of the rigorous solution (Supplemental Material [24], Sec. 3.2). Most importantly, this derivation clearly demonstrates that the magnitude of coupling which enters the effective photonic Hamiltonian is controlled by the signs of bianisotropy of the two adjacent disks and can be enhanced up to three times.

\section{EXPERIMENTAL VERIFICATION}

To confirm our prediction of Jackiw-Rebbi-type interface states, we have designed and tested experimentally a microwave prototype system based on high-index ceramic meta-atoms purchased from Ceramics Co. Ltd. having real and imaginary parts of permittivity equal to $\varepsilon^{\prime}=39$ and $\varepsilon^{\prime \prime}=0.004$, respectively. Each meta-atom is composed of two coaxial cylinders with different diameters $\left(d_{1}=29.1 \mathrm{~mm}\right.$, $\left.d_{2}=22.0 \mathrm{~mm}\right)$ and different heights $\left(h_{1}=9.0 \mathrm{~mm}\right.$ and $h_{2}=$ $3.0 \mathrm{~mm}$, respectively). The cylinders are placed on top of each other as illustrated in Figs. 1(a) and 1(b). Such design breaks the meta-atom mirror symmetry with respect to $O x y$ plane, which gives rise to magnetoelectric coupling. Parameters of cylinders were chosen to ensure that electric and magnetic dipole resonances appear in the same frequency range around $2.4-2.5 \mathrm{GHz}$.

Twelve fabricated ceramic particles were arranged in a linear array with the period $R=36 \mathrm{~mm}$. The structure was excited by two electric dipoles placed at two edges of the structure and separated by eight meta-atoms. The dipoles were connected to the port of vector network analyzer (VNA) Rohde \& Schwarz ZVB20 through the divider, which provided in-phase feeding. Near-field measurement was accomplished using automatic mechanical high precision scanner Trim TMC 3113 and subwavelength electric dipole connected to the second port of VNA which was used as the receiving antenna. To suppress multiple reflections, the measurements were carried out in an anechoic chamber with the walls covered by microwave absorbers Eccosorb VHP-12-NRL.

The measurements of the near-field map were performed for the two structures: the first structure with the same alignment of meta-atoms ("no wall" case) and the second one with half of the meta-atoms in the array flipped over, which ensured the opposite signs of magnetoelectric coupling $\mu$ for the two halves of the array ["wall" case, Fig. 1(a)]. In agreement with our theoretical predictions, we observe the interface states

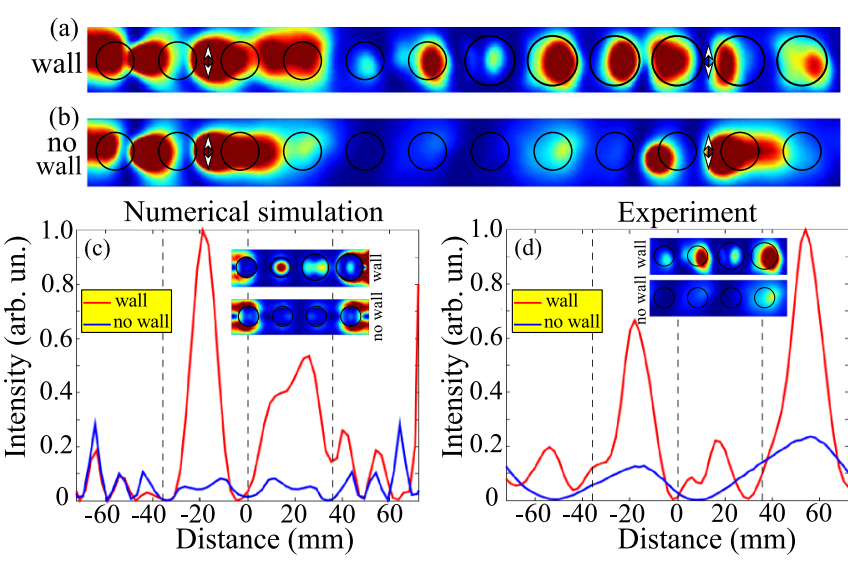

FIG. 5. Experimental observation of the topological interface state excited by the pair of electric dipoles in the array of 12 bianisotropic disks at frequency $f=2.435 \mathrm{GHz}$. (a), (b) Experimental near-field maps showing the quantity $I=\left|E_{x}\right|^{2}+\left|E_{y}\right|^{2}$ for the array (a) with and (b) without domain wall. White arrows show the location of the dipoles exciting the structure. (c), (d) Normalized intensity $I$ along the axis of the array according to (c) numerical simulation in CST MicROWAVE STUDIO software package and (d) experimental data. Distance is measured from the array center. Insets illustrate field distribution in the vicinity of array center for the cases with and without domain wall.

(Fig. 5) which manifest themselves through field localization in the vicinity of the domain wall.

Furthermore, our simulations suggest that the observed interface states are quite robust to disorder, whereas buckling of the array into zigzag geometry is accompanied by the topological transition from Jackiw-Rebbi interface states to the topological edge states (see Supplemental Material [24], Sec. 5 for details).

\section{DISCUSSION AND CONCLUSIONS}

To summarize, we have demonstrated an approach to tailor coupling constants in the effective photonic Hamiltonians by local modification of the disk bianisotropy. We believe that this finding bridges the existing gap between the need for tunable nonlinear couplings required for implementation of tunable topological states [27] from one side and experimental possibilities of nonlinear tuning allowing only for modification of on-site parameters. While at microwave frequencies, this problem is typically solved by insertion of varactor diodes [28-30], implementation of the same functionality in infrared or optical range remains highly challenging.

Besides that, we envision that our findings will initiate further research on photonic topological states in metamaterials with the staggered bianisotropy pattern which can be readily implemented experimentally but still wait for detailed theoretical investigations.

\section{ACKNOWLEDGMENTS}

We acknowledge valuable discussions with Dmitry Filonov. This work was supported by the Russian Foundation for Basic Research (Grants No. 18-32-20065 and No. 19- 
02-00939) and by the Ministry of Education and Science of the Russian Federation (Zadanie No. 3.2465.2017/4.6). Theoretical model for the interface states was developed with support by the Russian Science Foundation (Grant No.
18-72-00102). M.A.G. acknowledges partial support by the Foundation for the Advancement of Theoretical Physics and Mathematics "Basis." A.A.G. and D.V.Z. contributed equally to this work.
[1] A. B. Evlyukhin, S. M. Novikov, U. Zywietz, R. L. Eriksen, C. Reinhardt, S. I. Bozhevolnyi, and B. N. Chichkov, Demonstration of magnetic dipole resonances of dielectric nanospheres in the visible region, Nano Lett. 12, 3749 (2012).

[2] A. I. Kuznetsov, A. E. Miroshnichenko, Y. H. Fu, J. B. Zhang, and B. Luk'yanchuk, Magnetic light, Sci. Rep. 2, 492 (2012).

[3] S. Jahani and Z. Jacob, All-dielectric metamaterials, Nat. Nanotechnol. 11, 23 (2016).

[4] A. I. Kuznetsov, A. E. Miroshnichenko, M. L. Brongersma, Y. S. Kivshar, and B. Luk'yanchuk, Optically resonant dielectric nanostructures, Science 354, aag2472 (2016).

[5] A. E. Krasnok, A. E. Miroshnichenko, P. A. Belov, and Y. S. Kivshar, All-dielectric optical nanoantennas, Opt. Express 20, 20599 (2012).

[6] M. R. Shcherbakov, D. N. Neshev, B. Hopkins, A. S. Shorokhov, I. Staude, E. V. Melik-Gaykazyan, M. Decker, A. A. Ezhov, A. E. Miroshnichenko, I. Brener, A. A. Fedyanin, and Y. S. Kivshar, Enhanced third-harmonic generation in silicon nanoparticles driven by magnetic response, Nano Lett. 14, 6488 (2014).

[7] A. B. Khanikaev, S. H. Mousavi, W. K. Tse, M. Kargarian, A. H. MacDonald, and G. Shvets, Photonic topological insulators, Nat. Mater. 12, 233 (2013).

[8] A. Slobozhanyuk, S. H. Mousavi, X. Ni, D. Smirnova, Y. S. Kivshar, and A. B. Khanikaev, Three-dimensional all-dielectric photonic topological insulator, Nat. Photon. 11, 130 (2017).

[9] L. Lu, J. D. Joannopoulos, and M. Soljačić, Topological photonics, Nat. Photon. 8, 821 (2014).

[10] L. Lu, J. D. Joannopoulos, and M. Soljačić, Topological states in photonic systems, Nat. Phys. 12, 626 (2016).

[11] A. B. Khanikaev and G. Shvets, Two-dimensional topological photonics, Nat. Photon. 11, 763 (2017).

[12] T. Ozawa, H. M. Price, A. Amo, N. Goldman, M. Hafezi, L. Lu, M. C. Rechtsman, D. Schuster, J. Simon, O. Zilberberg, and I. Carusotto, Topological photonics, Rev. Modern Phys. 91, 015006 (2019).

[13] A. P. Slobozhanyuk, A. B. Khanikaev, D. S. Filonov, D. A. Smirnova, A. E. Miroshnichenko, and Y. S. Kivshar, Experimental demonstration of topological effects in bianisotropic metamaterials, Sci. Rep. 6, 22270 (2016).

[14] A. Slobozhanyuk, A. V. Shchelokova, X. Ni, S. H. Mousavi, D. A. Smirnova, P. A. Belov, A. Alú, Y. S. Kivshar, and A. B. Khanikaev, Near-field imaging of spin-locked edge states in all-dielectric topological metasurfaces, Appl. Phys. Lett. 114, 031103 (2019).

[15] Y. Yang, Z. Gao, H. Xue, L. Zhang, M. He, Z. Yang, R. Singh, Y. Chong, B. Zhang, and H. Chen, Realization of a three-dimensional photonic topological insulator, Nature $\mathbf{5 6 5}$, 622 (2019).

[16] W. Tan, Y. Sun, H. Chen, and S.-Q. Shen, Photonic simulation of topological excitations in metamaterials, Sci. Rep. 4, 3842 (2014).

[17] D. G. Angelakis, P. Das, and C. Noh, Probing the topological properties of the Jackiw-Rebbi model with light, Sci. Rep. 4, 6110 (2014).

[18] T. X. Tran and F. Biancalana, Linear and nonlinear photonic Jackiw-Rebbi states in interfaced binary waveguide arrays, Phys. Rev. A 96, 013831 (2017).

[19] E. M. Purcell and C. R. Pennypacker, Scattering and absorption of light by nonspherical dielectric grains, Astrophys. J. 186, 705 (1973).

[20] B. T. Draine, The discrete-dipole approximation and its application to interstellar graphite grains, Astrophys. J. 333, 848 (1988).

[21] A. B. Evlyukhin, C. Reinhardt, A. Seidel, B. S. Luk'yanchuk, and B. N. Chichkov, Optical response features of Sinanoparticle arrays, Phys. Rev. B 82, 045404 (2010).

[22] A. B. Evlyukhin, C. Reinhardt, and B. N. Chichkov, Multipole light scattering by nonspherical nanoparticles in the discrete dipole approximation, Phys. Rev. B 84, 235429 (2011).

[23] L. Novotny and B. Hecht, Principles of Nano-Optics (Cambridge University Press, Cambridge, 2006).

[24] See Supplemental Material at http://link.aps.org/supplemental/ 10.1103/PhysRevB.99.205122 for the detailed derivation of the effective photonic Hamiltonian, analysis of its bulk spectrum, associated interface states and their robustness with respect to disorder.

[25] M. O. Goerbig, J.-N. Fuchs, G. Montambaux,, and F. Piéchon, Tilted anisotropic Dirac cones in quinoid-type graphene and $\alpha-(\text { BEDT-TTF })_{2} I_{3}$, Phys. Rev. B 78, 045415 (2008).

[26] S.-Q. Shen, Topological Insulators. Dirac Equation in Condensed Matters (Springer, Berlin, 2012).

[27] Y. Hadad, A. B. Khanikaev, and A. Alu, Self-induced topological transitions and edge states supported by nonlinear staggered potentials, Phys. Rev. B 93, 155112 (2016).

[28] Y. Hadad, J. C. Soric, A. B. Khanikaev, and A. Alú, Selfinduced topological protection in nonlinear circuit arrays, Nat. Electron. 1, 178 (2018).

[29] D. A. Dobrykh, A. V. Yulin, A. P. Slobozhanyuk, A. N. Poddubny, and Yu. S. Kivshar, Nonlinear Control of Electromagnetic Topological Edge States, Phys. Rev. Lett. 121, 163901 (2018).

[30] M. Serra-Garcia, R. Süsstrunk, and S. D. Huber, Observation of quadrupole transitions and edge mode topology in an LC circuit network, Phys. Rev. B 99, 020304 (2019). 